\title{
Specific binding of the regulatory protein ExpG to promoter regions of the galactoglucan biosynthesis gene cluster of Sinorhizobium meliloti - a combined molecular biology and force spectroscopy investigation
}

\author{
Frank Wilco Bartels, ${ }^{\mathrm{a}, 1}$ Birgit Baumgarth, ${ }^{\mathrm{b}, 1}$ Dario Anselmetti, ${ }^{\mathrm{a}}$ Robert Ros, ${ }^{\mathrm{a}, *}$ \\ and Anke Becker ${ }^{\mathrm{b}}$ \\ ${ }^{a}$ Experimentelle Biophysik, Fakultät für Physik, Universität Bielefeld, 33615 Bielefeld, Germany \\ ${ }^{\mathrm{b}}$ Lehrstuhl für Genetik, Fakultät für Biologie, Universität Bielefeld, 33615 Bielefeld, Germany
}

Received 24 February 2003, and in revised form 14 June 2003

\begin{abstract}
Specific protein-DNA interaction is fundamental for all aspects of gene transcription. We focus on a regulatory DNA-binding protein in the Gram-negative soil bacterium Sinorhizobium meliloti 2011, which is capable of fixing molecular nitrogen in a symbiotic interaction with alfalfa plants. The ExpG protein plays a central role in regulation of the biosynthesis of the exopolysaccharide galactoglucan, which promotes the establishment of symbiosis. ExpG is a transcriptional activator of exp gene expression. We investigated the molecular mechanism of binding of ExpG to three associated target sequences in the exp gene cluster with standard biochemical methods and single molecule force spectroscopy based on the atomic force microscope (AFM). Binding of ExpG to $\exp A 1, \exp G-\exp D 1$, and $\exp E 1$ promoter fragments in a sequence specific manner was demonstrated, and a 28 bp conserved region was found. AFM force spectroscopy experiments confirmed the specific binding of ExpG to the promoter regions, with unbinding forces ranging from 50 to $165 \mathrm{pN}$ in a logarithmic dependence from the loading rates of $70-79000 \mathrm{pN} / \mathrm{s}$. Two different regimes of loading rate-dependent behaviour were identified. Thermal off-rates in the range of $k_{\text {off }}=(1.2 \pm 1.0) \times 10^{-3} \mathrm{~s}^{-1}$ were derived from the lower loading rate regime for all promoter regions. In the upper loading rate regime, however, these fragments exhibited distinct differences which are attributed to the molecular binding mechanism.
\end{abstract}

Keywords: Atomic force microscopy; Galactoglucan; Protein-DNA interaction; Regulatory protein; Single molecule force spectroscopy; Sinorhizobium meliloti

\section{Introduction}

The soil bacterium Sinorhizobium meliloti is capable of fixing molecular nitrogen in a symbiotic interaction with plants of the genera Medicago, Melilotus, and Trigonella. It has the ability to produce two acidic exopolysaccharides (EPSs), succinoglycan (EPS I) and galactoglucan (EPS II). EPS I is required for invasion of Medicago sativa root nodules by $S$. meliloti, but can be

\footnotetext{
* Corresponding author. Fax: +49-521-106-2959.

E-mail address: Robert.Ros@physik.uni-bielefeld.de (R. Ros).

${ }^{1}$ F.W.B. and B.B. contributed equally to this work.
}

replaced by EPS II (Glazebrook and Walker, 1989; Gonzalez et al., 1996; Wang et al., 1999). EPS II consists of alternating glucose and galactose residues which are decorated by acetyl and pyruvyl groups (Her et al., 1990). Biosynthesis of EPS II is directed by the $30 \mathrm{~kb}$ exp gene cluster that comprises 22 genes organized in four operons (Becker et al., 1997; Rüberg et al., 1999).

Under standard culture conditions in a complex medium, wild type strain S. meliloti 2011 produces EPS I and synthesizes only traces of EPS II, but phosphatelimiting conditions stimulate the production of this EPS (Zhan et al., 1991). Production of EPS II was also observed in the presence of a mutation in either of the 
regulatory genes $\exp R$ (Glazebrook and Walker, 1989; Pellock et al., 2002) or mucR (Keller et al., 1995; Zhan et al., 1989) that are unlinked to the exp gene cluster. An additional regulatory gene, $\exp G(m u c S)$, was identified in the exp gene cluster (Astete and Leigh, 1996; Becker et al., 1997; Rüberg et al., 1999). Extra copies of $\exp G$ stimulate transcription of the $\exp A, \exp D$, and $\exp E$ operons (Rüberg et al., 1999). Furthermore, $\exp G$ is required for the stimulation of these operons under phosphate-limiting conditions indicating that ExpG acts as a transcriptional activator of exp gene expression (Astete and Leigh, 1996; Rüberg et al., 1999). An identical role was suggested for the mucS gene of the halotolerant $S$. meliloti strain EFB1 constitutively producing EPS I and EPS II (Lloret et al., 1998, 2002).

ExpG shows homologies to transcriptional regulators of the MarR family (Becker et al., 1997). These regulators bind DNA through a helix-turn-helix (HTH) motif (Cohen et al., 1993; Sulavik et al., 1995). Members of the MarR family control an assortment of biological functions including the expression of resistance to multiple antibiotics, detergents and oxidative stress agents, organic solvents, and pathogenic factors (Alekshun and Levy, 1999; Miller and Sulavik, 1996). Most members of the MarR family are repressors and only few act as activators (Egland and Harwood, 1999; Komeda et al., 1996; Oscarsson et al., 1996). The homology of ExpG to regulators of the MarR family implies that ExpG itself exerts the positive regulation of exp gene expression by binding to promoter regions in the exp gene cluster.

To confirm and characterize the binding of the regulatory protein ExpG to DNA, we performed experiments not only in ensembles - a standard in molecular biology_but also on single molecules. During the last decade, the development of single molecule methods allowed direct measurement of intermolecular forces between individual biomolecular binding partners (Bustamante et al., 2000). Especially, atomic force microscopy (AFM) pioneered measurement of the unbinding forces of single bound complexes in the picoNewton force range, p.e., between complementary DNA strands (Lee et al., 1994a; Strunz et al., 1999) or single ligand receptor pairs (Dammer et al., 1995; Florin et al., 1994; Fritz et al., 1998; Hinterdorfer et al., 1996; Lee et al., 1994b; Ros et al., 1998). A spatial resolution in the nanometer range is an additional advantage in AFM force measurements (Ros et al., 1998). The measured separation forces, however, depend on the loading rate that is exerted on the bound complex, i.e., the time dependency of the external forces (loading rate $=\mathrm{AFM}$ retract velocity $\times$ molecule elasticity). A slowly increasing load provides ample time for thermal fluctuations to drive the system over the energy barrier of the binding potential, resulting in small unbinding forces (Evans and Ritchie, 1997). A logarithmic dependence of the forces on the loading rate was found in systems close to ther- modynamical equilibrium, where a single energy barrier governs the separation process (Carrion-Vasquez et al., 1999; Fritz et al., 1998; Rief et al., 1997; Schwesinger et al., 2000). In contrast, force spectroscopy on biotin/ avidin and biotin/streptavidin complexes revealed the influence of multiple energy barriers on the unbinding mechanism of these systems (De Paris et al., 2000; Merkel et al., 1999).

In this paper, we strive to (i) show that the ExpG protein binds to promoter regions in the galactoglucan biosynthesis (exp) gene cluster, (ii) confirm the specificity of this interaction, and (iii) characterize the binding mechanism involved with respect to its thermal off-rate and additional molecular parameters describing the energy landscape of the separation process. Thereby, single molecule AFM force spectroscopy contributes information regarding protein-DNA binding that complements and exceeds the data gained from standard molecular biology experiments.

\section{Methods}

\subsection{Bacterial strains, plasmids, and growth conditions}

Escherichia coli M15 [pRep4] (Gottesman et al., 1981), used as expression strain, was cultivated in SB (Super broth) medium $(32 \mathrm{~g} / \mathrm{L}$ tryptone, $20 \mathrm{~g} / \mathrm{L}$ yeast extract, and $5 \mathrm{~g} / \mathrm{L} \mathrm{NaCl}$ adjusted to $\mathrm{pH} 7.6$ ) at $37^{\circ} \mathrm{C}$. Plasmid pHisG4032 was constructed by insertion of a $573 \mathrm{bp}$ BamHI-HindIII fragment carrying $\exp G$ of S. meliloti 2011 (Casse et al., 1979) into vector pWH844 (Schirmer et al., 1997). This Fragment was amplified by PCR with primer HisG5 (5'-AAAAGGATCCAT GAACCACAGGATACTCTATCCGT, BamHI restriction site is underlined) and HisG3 (5'-AAAA AAGCTTCATGTTACCGCCCTTGCTCG, HindIII restriction site is underlined) from pARIIa (Becker et al., 1997). Expression of the hybrid gene resulted in a fusion protein consisting of ExpG from amino acid one (Met) to amino acid 191 (Ile), with an (His) 6 GlySer $\mathrm{N}$-terminal tag.

\subsection{Proteins}

Expression of recombinant (His) ${ }_{6}$ ExpG fusion protein was performed essentially as described (Becker et al., 2001). Purification was carried out by Ni-NTA affinity chromatography (Qiagen). Purified fusion protein was concentrated using an Ultrafree 4 centrifugal concentrator (Millipore), resuspended in a buffer $(250 \mathrm{mM} \mathrm{NaCl}, 10 \mathrm{mM}$ Tris, $1 \mathrm{mM}$ DTT, and $50 \%$ glycerol) and stored at $-20^{\circ} \mathrm{C}$. The concentration of purified protein was determined by using the Bio-Rad Protein Assay (Bio Rad Laboratories, München; Bradford, 1976). 


\subsection{DNA fragments}

DNA fragments for Electrophoretic Mobility Shift Assays (EMSAs) and AFM experiments were amplified by PCR. Primers used for PCR, length and labeling of resulting DNA fragments are summarized in Table 1. pARIIa (Becker et al., 1997) was used as template for amplification of fragments I, Ia and II whereas pARIV (Becker et al., 1997) was applied as template for amplification of fragments III and IIIa. Competitor fragments were obtained using unlabeled primers.

\subsection{EMSA analysis}

Cy3-labeled DNA fragments I, Ia, II, III, or IIIa (Table 1) in various concentrations were mixed with purified (His) ${ }_{6} \operatorname{ExpG}(0.125 \mu \mathrm{g} / \mu \mathrm{l})$ in a reaction buffer containing $50 \mathrm{mM}$ Tris- $\mathrm{HCl}, \mathrm{pH} 8.0,100 \mathrm{mM} \mathrm{NaCl}$, $0.1 \mathrm{mM} \quad \mathrm{MgSO}_{4}, 5 \%$ glycerol, $0.05 \mathrm{mg} / \mathrm{ml}$ sonicated hering testes DNA and $0.5 \mathrm{mg} / \mathrm{ml}$ bovine serum albumin and were subjected to EMSAs (Bertram-Drogatz et al., 1997; Bertram-Drogatz et al., 1998). In competition assays, protein was added to Cy3-labeled DNA fragments in the presence of various concentrations of competitor DNA fragments. After incubation at $20^{\circ} \mathrm{C}$ for $15 \mathrm{~min}$, the reaction was loaded onto a $2 \%$ non-denaturing agarose gel prepared in gel buffer $(40 \mathrm{mM}$ Tris base, $10 \mathrm{mM}$ sodium acetate, and $1 \mathrm{mM}$ EDTA adjusted to $\mathrm{pH} 7.8$ with acetic acid) and electrophoresed at $4{ }^{\circ} \mathrm{C}$ in gel buffer at $4.5 \mathrm{~V} / \mathrm{cm}$ for $2 \mathrm{~h}$. Gel images were acquired using a Typhoon 8600 Variable Mode Imager (Amersham Bioscience).

\subsection{Sample surface and AFM tip modification}

For AFM measurements, $\mathrm{Si}_{3} \mathrm{~N}_{4}$ cantilevers (Microlever, Thermomicroscopes, Sunnyvale, CA, USA) were first activated by dipping for $10 \mathrm{~s}$ in concentrated nitric acid and silanized in a solution of $2 \%$ aminopropyltri- ethoxysilane (Sigma) in dry toluene for $2 \mathrm{~h}$. After washing with toluene, the cantilevers were incubated with $1 \mathrm{mM} N$-hydroxysuccinimide-poly(ethylene glycol)-maleimide (Shearwater Polymers, Huntsville, AL, USA) in $0.1 \mathrm{M}$ potassium phosphate buffer, $\mathrm{pH} 8.0$, for $30 \mathrm{~min}$ at room temperature. After washing with phosphate buffer, the cantilevers were incubated with $10 \mathrm{ng} / \mu \mathrm{l}$ of the respective DNA target sequence (see above) bearing a sulfhydryl label (Table 1) in binding buffer solution $(50 \mathrm{mM}$ Tris- $\mathrm{HCl}, 100 \mathrm{mM} \mathrm{NaCl}, 0.1 \mathrm{mM}$ $\mathrm{NiCl}_{2}$, and $\mathrm{pH} 8.3$ ) overnight at $4{ }^{\circ} \mathrm{C}$. The cantilevers were washed with binding buffer and used for force spectroscopy experiments. Modified tips were usable for at least one week if stored at $4{ }^{\circ} \mathrm{C}$.

Mica surfaces (Provac AG, Balzers, Liechtenstein) were silanized with aminopropyltriethoxysilane in an exsiccator (Lyubchenko et al., 1993) and incubated with $4 \mu \mathrm{M}$ (His) ${ }_{6}$ ExpG protein and $20 \mu \mathrm{M}$ bis(sulfosuccinimidyl)suberate-sodium salt (Sigma) in $0.1 \mathrm{M}$ potassium phosphate buffer, $\mathrm{pH} 7.5$, for $1 \mathrm{~h}$ at $4^{\circ} \mathrm{C}$. The sample was washed with binding buffer afterwards. Modified surfaces were stable for at least 2 days if stored at $4{ }^{\circ} \mathrm{C}$. A scheme of the experimental setup is shown in Fig. 2.

\subsection{Dynamic force spectroscopy}

Force spectroscopy measurements were performed with a commercial AFM (Multimode, Veeco Instruments, Santa Barbara, CA, USA) at $25^{\circ} \mathrm{C}$. Acquisition of the cantilever deflection force signal and the vertical movement of the piezo electric elements was controlled by a 16 bit AD/DA card (PCI-6052E, National Instruments, Austin, TX, USA) and a high-voltage amplifier $(600 \mathrm{H}$, NanoTechTools, Echandens, Switzerland) via a home-built software based on Labview (National Instruments). The deflection signal was low pass filtered $(<6 \mathrm{kHz})$ and box averaged by a factor of 10 , giving a typical experimental data set of 2000 points per forcedistance curve.

Table 1

Primers and labeling used for PCR of the DNA fragments for EMSAs and AFM experiments

\begin{tabular}{|c|c|c|c|c|}
\hline Promoter region & Primer no. & Primer sequence $\left(5^{\prime}-3^{\prime}\right)$ & $\mathrm{bp}$ & Fragment \\
\hline $\exp A 1$ & $\begin{array}{l}\mathrm{A} 1^{\mathrm{b}} \\
\mathrm{A} 4^{\mathrm{a}}\end{array}$ & $\begin{array}{l}\text { CATCAACTCTTGCACGCAGC } \\
\text { CAGAACGGTCGAACAGAGGT }\end{array}$ & 301 & I \\
\hline $\exp A 1$ & $\begin{array}{l}\mathrm{A} 3 \\
\mathrm{~A} 4^{\mathrm{a}}\end{array}$ & $\begin{array}{l}\text { GCACAACAATAGATTCTTGG } \\
\text { CAGAACGGTCGAACAGAGGT }\end{array}$ & 176 & Ia \\
\hline $\exp G$ & $\begin{array}{l}\mathrm{G} 1^{\mathrm{b}} \\
\mathrm{G} 4^{\mathrm{a}}\end{array}$ & $\begin{array}{l}\text { TCTCCATTGGGAACGTACTT } \\
\text { CTGGTCCAGATTCACTCAAA }\end{array}$ & 158 & II \\
\hline $\exp E 1$ & $\begin{array}{l}\mathrm{E} 1^{\mathrm{a}+\mathrm{b}} \\
\mathrm{E} 5\end{array}$ & $\begin{array}{l}\text { AAAGTGGCCATCTGCTTCTC } \\
\text { CAGTCTCCGACAGTTTCAAC }\end{array}$ & 326 & III \\
\hline $\exp E 1$ & $\begin{array}{l}\text { E3 } \\
\text { E5 }\end{array}$ & $\begin{array}{l}\text { AAACATATCTCCATGCTGTG } \\
\text { CAGTCTCCGACAGTTTCAAC }\end{array}$ & 171 & IIIa \\
\hline
\end{tabular}

\footnotetext{
${ }^{\mathrm{a}} 5^{\prime} \mathrm{Cy} 3$-labeled for EMSAs.

${ }^{\mathrm{b}}{ }^{\prime} \mathrm{SH}$-labeled for AFM experiments.
} 
The spring constants of all AFM cantilevers were calibrated by the thermal fluctuation method (Hutter and Bechhoefer, 1993) with an absolute uncertainty of approximately $15 \%$. Spring constants of the cantilevers used ranged from 13 to $22 \mathrm{pN} / \mathrm{nm}$.

For loading rate-dependent measurements, the retract velocity of the piezo was varied while keeping the approach velocity constant. The measured force-distance curves were analyzed with a Matlab program (MathWorks, Natick, MA, USA) and corrected to display the actual molecular distances calculated from the $\mathrm{z}$ piezo extension. This correction is especially important for soft cantilevers. To obtain the loading rate, the retract velocity was then multiplied by the elasticity of the molecular system which was determined from the slope of the corrected force-distance curves on the last 20 data points before the unbinding events.

\section{Results and discussion}

\section{1. $\operatorname{Exp} G$ binds to $\operatorname{expA1,} \exp G-\operatorname{expD1}$, and $\exp E 1$ promoter regions}

ExpG binding to promoter regions in the exp gene cluster was demonstrated with EMSA. For this purpose a (His) ${ }_{6}$ ExpG fusion protein was expressed and purified by Ni-NTA affinity chromatography. SDS-PAGE of the purified protein exhibited a major band migrating at approximately $22 \mathrm{kDa}$ which was detected using antiHis-tag antibody and corresponds to the calculated MW of $22.3 \mathrm{kDa}$ (data not shown).

Fragments comprising the intergenic regions between $\exp C$ and $\exp A 1, \exp G$ and $\operatorname{expD1}$ as well as $\operatorname{expD2}$ and expE1 (Table 1, Fig. 1A) were chosen for these (His) ${ }_{6}$ ExpG-DNA binding experiments, since extra copies of $\exp G$ resulted in enhanced transcription of the $\exp A, \exp D$, and $\exp E$ operons (Rüberg et al., 1999). Alignment of these intergenic regions exhibits a $28 \mathrm{bp}$ conserved domain containing a palindromic sequence (Fig. 1B) that may function as an ExpG binding site. The palindrome contains an AT-rich axis, a characteristic feature of typical binding sites for HTH-proteins like ExpG (Becker et al., 1997). This conserved region also shows high homologies to the muc boxes in $S$. meliloti EFB1 which were suggested as targets for the MucS protein (Lloret et al., 2002).

(His) ${ }_{6}$ ExpG reduced the electrophoretic mobility of fragments I, II, and III (Fig. 1) indicating the binding of ExpG to the $\exp A 1, \exp G-\operatorname{expD1}$, and $\operatorname{expE1}$ promoter regions. Shorter fragments of the expAI and expEI promoter regions (fragment Ia and IIIa, Table 1) containing the conserved region were subjected to EMSAs to further restrict the DNA binding site of ExpG

A

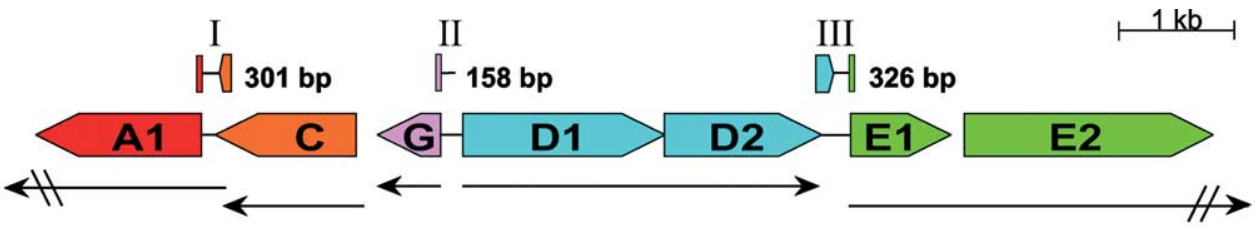

B

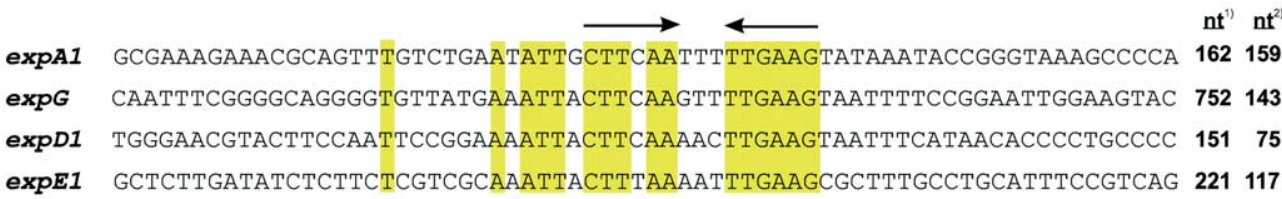

C competitor:

$\exp A$ (fragment I) $\exp D G$ (fragment II)

$\exp E$ (fragment III)

$\operatorname{expA}$ (fragment la)

$\exp E$ (fragment IIla)
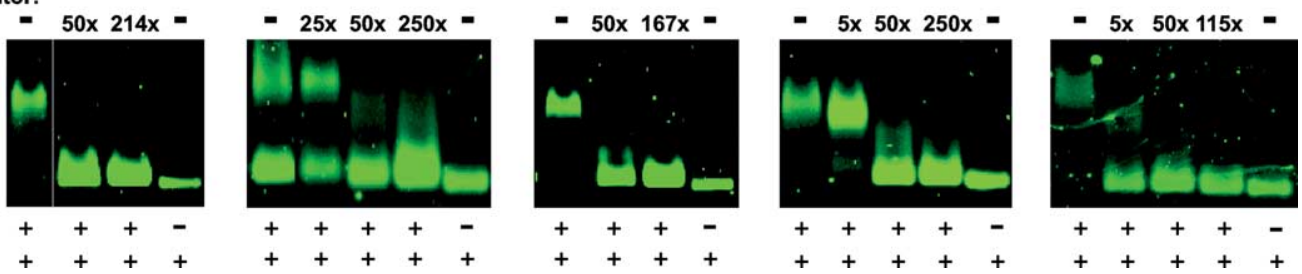

Fig. 1. (A) exp gene region from $\exp A 1$ to $\operatorname{expE2}(10.330 \mathrm{nt})$ from the exp gene cluster of $S$. meliloti 2011 (Casse et al., 1979). Transcriptional units are marked by arrows. I, II, and III are DNA fragments for EMSAs and AFM experiments. (B) Alignment of the conserved region found in the promoter regions of $\exp A 1, \exp G, \exp D 1$, and $\exp E 1$. Residues identical in the four promoter regions are boxed. Inverted arrows indicate a palindrome found within the conserved region. ${ }^{1)}$ Distance of the last boxed nucleotide to ATG of the first open reading frame in each operon. ${ }^{2)}$ Distance to the $5^{\prime}$ end of the DNA fragments I, II, or III. (C). EMSAs with purified (His) ${ }_{6}$ ExpG fusion protein, DNA fragments I, II, III, Ia, or IIIa and specific competitor DNA fragments. Protein was added to all reactions to give a final protein concentration of $0.125 \mu \mathrm{g} / \mu \mathrm{l}$. Unlabeled competitor DNA was added in increasing amounts from 5-fold to 250-fold excess over the Cy3-labeled DNA fragments. 

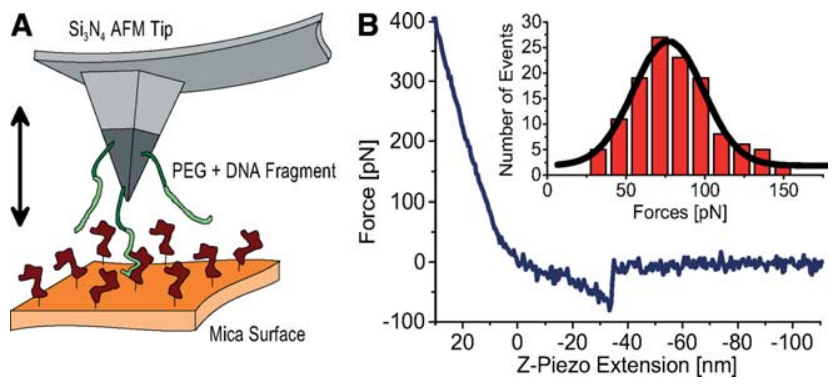

Fig. 2. Force spectroscopy measurements. (A) The experimental setup consists of a $\mathrm{Si}_{3} \mathrm{~N}_{4}$ AFM tip with DNA fragments attached via poly(ethylene glycol) spacer molecules and a flat mica surface on which the (His) ${ }_{6}$ ExpG proteins are immobilized. Cycling the tip and sample between approach and retract with constant velocities results in a series of force-distance measurements. (B) A typical force-distance curve (only retractive part displayed). An unbinding event can be identified by a certain distance from the point of contact due to the length of the polymer linker and the stretching of this linker directly prior to the point of bond rupture. Rupture forces of a given series are combined to form a histogram (B, small inset) with an almost Gaussian distribution. The mean value of the Gaussian equals the most probable unbinding force, with statistical errors given by standard deviation $\left(2 \sigma / N^{-1 / 2}\right.$ for $95.4 \%$ confidence level).
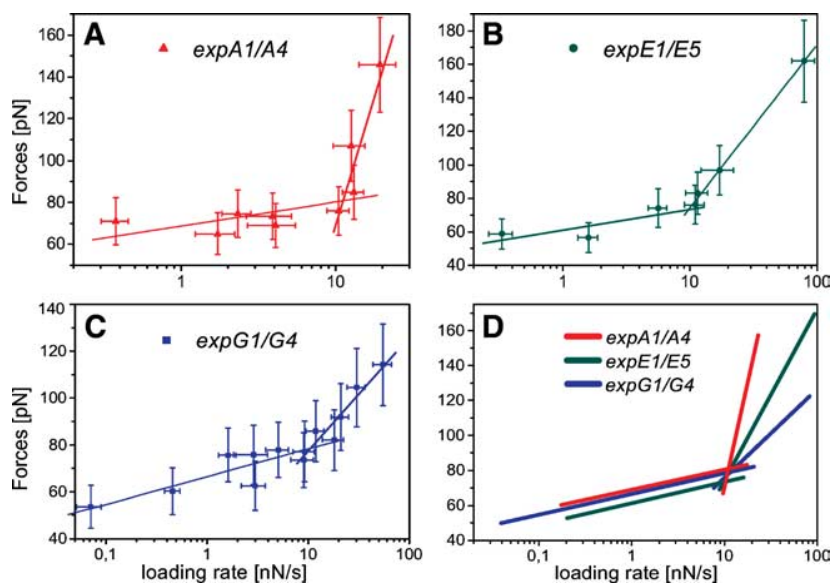

Fig. 4. Dynamic force spectroscopy. Loading rate dependent measurements of the unbinding forces are displayed for complexes formed by the (His) $)_{6}$ ExpG protein and each of its three DNA target sequences (A-C). Two regions can be distinguished in every dataset. In the higher loading rate region, when each dataset is subjected to a linear fit, the slopes differ widely from each other (see D for comparison of the fits), and the individual DNA fragment in use can be identified by this behavior. In the lower loading rate region, however, the different protein-DNA complexes share a single slope under a linear fit within the error margin. This corresponds to a joint natural thermal off-rate of $k_{\text {off }}=(1.2 \pm 1.0) \times 10^{-3} \mathrm{~s}^{-1}$, derived by extrapolating the line fit to the state of zero external force.

(Fig. 1C). Fragments Ia and IIIa were retarded by (His) ${ }_{6}$ ExpG supporting the assumption that the conserved region might be involved in binding of ExpG, which has to be fortified by further analyses. The results of these EMSA experiments together with previous reports (Rüberg et al., 1999) suggest that ExpG exerts a stimulating effect on exp gene expression by binding to
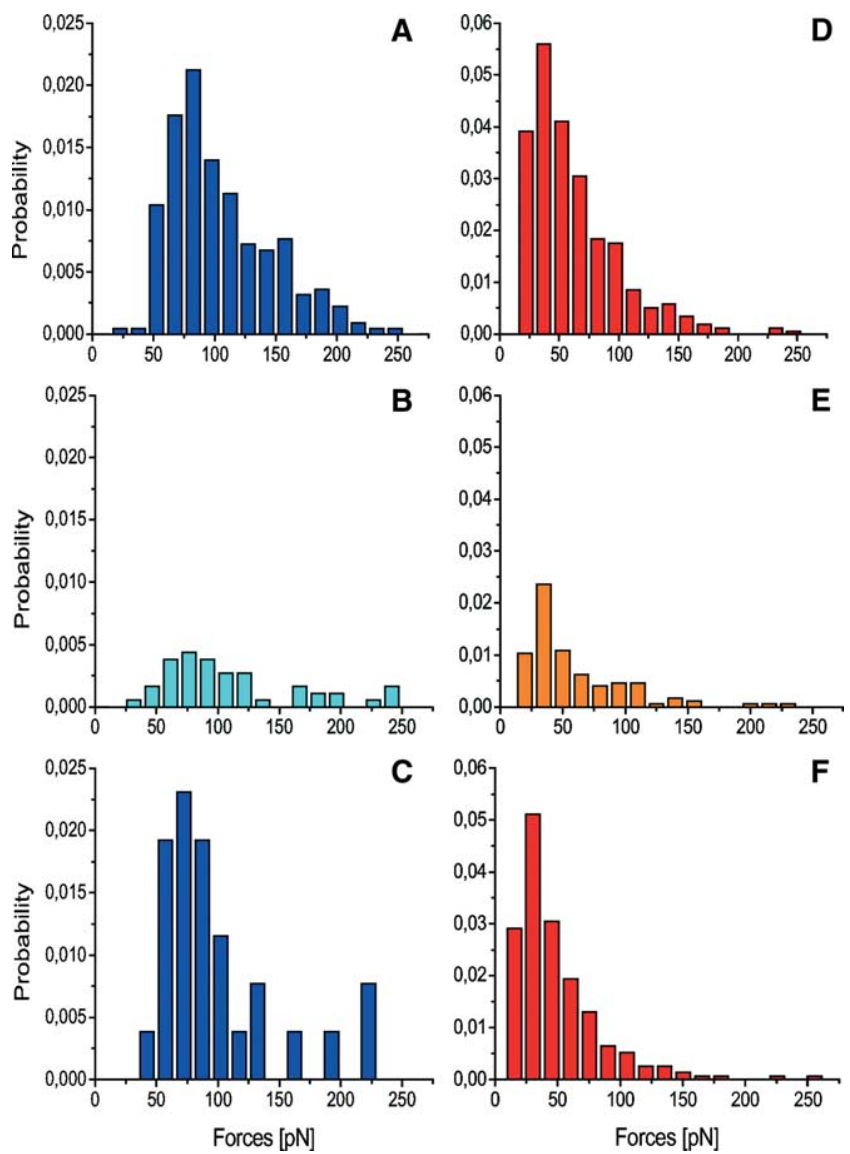

Fig. 3. Single molecule competition experiments. Force spectroscopy measurements were performed under alternating buffer conditions with a single tip and sample system for each series. (A-C) In this series, the $\exp A 1 / A 4$ DNA fragment was attached to the tip. Unbinding events were first measured in the original binding buffer solution (A). When free $\exp A 1 / A 4$ fragments were inserted into the buffer at an excess of $60: 1$, the total unbinding probability was reduced (B). By exchanging the buffer back to the original conditions, the system was reactivated after $20 \mathrm{~min}(\mathrm{C})$. (D-F) A series with the $\exp G 1 / G 4$ fragment bound to the tip reveals a reduction in unbinding probability from the original binding buffer (D) to the application of free (His) ${ }_{6}$ ExpG protein as a competitor in a ratio of 1:1 (E). By a buffer exchange back to the original buffer solution, the system was reactivated after $85 \mathrm{~min}(\mathrm{~F})$.

the promoter regions of the $\exp A, \exp D, \exp G$, and $\exp E$ operons.

\subsection{ExpG binds specifically to exp promoter regions}

To investigate the protein-DNA interaction on a single molecule basis, the binding partners were covalently bound to the AFM tip and to the sample surface, respectively. The DNA fragment was attached to the tip via a polymer spacer analogous to (Strunz et al., 1999) while the (His) ${ }_{6}$ ExpG protein was immobilized on the surface by a short linker molecule coupled to one of the five ExpG lysines (Becker et al., 1997) (Fig. 2A). When the tip was approached to and retracted from the surface, the flexibility of the polymer chain allowed the 
DNA molecules to access the binding pockets of immobilized proteins. By plotting the force acting on the AFM tip against the vertical position (given by the extension of the piezo), unbinding events can be identified by a characteristical stretching of the polymer spacer before the point of bond rupture (where the tip snaps back to zero force). A typical force-distance curve is shown in Fig. 2B. The rupture forces from multiple approach-retract cycles under a single retract velocity were combined in a histogram. The mean value of the nearly Gaussian distribution was taken as the most probable rupture force, its error given by standard deviation and the uncertainty of the spring constant.

The total unbinding probability (events/cycles) usually amounted to $15 \%$. (This was checked against the background signal, i.e., a series of measurements with a functionalized AFM tip and a surface without the protein but prepared as normal in all other respects, to reveal no unbinding events.) When free DNA fragments were added to the buffer solution in an excess of appr. 60:1 regarding the proteins, a distinct reduction in unbinding probability was observed for all three DNA target sequences (shown in Figs. 3A-C for the expA1/A4 fragment). Moreover, when both tip and sample were washed with buffer solution and reinstalled with the original, competitor-free buffer in place, the system could be reactivated to almost its full former unbinding probability. The distinct influence of the competitor fragments clearly indicates the specificity of the binding process.

In an experiment with the $\exp G 1 / G 4$ DNA fragment II (Table 1), free (His) ${ }_{6}$ ExpG proteins were added to the buffer as an alternative competitor. Even at a ratio as small as appr. 1:1 regarding the DNA density on the tip (which was calculated based on measurements for PEGbound ligands on aminosilane monolayers by Hinterdorfer et al., 1998), a distinct reduction in unbinding probability was observed. Again, the system could be reactivated to almost its former unbinding probability when buffers are changed back to the original conditions (Figs. 3D-F).

To confirm the results from the AFM experiments demonstrating specific binding of ExpG to the expAl, $\exp G-\exp D$, and $\operatorname{expE1}$ promoter regions, binding reactions were carried out in the presence of unlabeled specific competitor DNA fragments and analyzed in EMSAs (Fig. 1C). Labeled fragments I, II, III, and Ia were not retarded by (His) ${ }_{6}$ ExpG in the presence of a 50 -fold excess of unlabeled competitor fragments I, II, III, and Ia, respectively. Competition for binding of (His) ${ }_{6}$ ExpG to labeled fragment IIIa was effective at a 5fold excess of unlabeled fragment IIIa. EMSAs with non-specific competitor DNA fragments derived from the Epstein-Barr virus (Rawlins et al., 1985) in increasing concentration had virtually no effect on the binding of (His) ${ }_{6}$ ExpG to the specific DNA fragments and thus confirm the specificity of binding (data not shown). Results from EMSA and AFM experiments using specific DNA competitor fragments were consistent. This demonstrates that measuring unbinding forces by AFM force spectroscopy is a suitable technique to determine the specificity of binding of a protein to DNA.

\subsection{Molecular interaction parameters}

To obtain thermodynamical and structural information concerning the binding, we measured the most probable unbinding forces for the three DNA-protein complexes in dependence on the loading rate by varying the retract velocity. For each DNA target sequence, typically $150-300$ unbinding events (from 1000 to 2000 approach/retract cycles) were recorded at 7-9 different retract velocities ranging from 10 to $8000 \mathrm{~nm} / \mathrm{s}$, while the approach velocity was kept constant at $1000 \mathrm{~nm} / \mathrm{s}$. These resulted in loading rates in the range from 70 to $79000 \mathrm{pN} / \mathrm{s}$.

The results for the three DNA target sequences are shown in Fig. 4. When the unbinding forces are plotted against the corresponding loading rates on a logarithmic scale, two regions with different slopes emerge for all DNA fragments. In both regions the experimental data can be fitted to a linear function according to the formula given by Strunz et al. (1999)

$F=\frac{k_{\mathrm{B}} T}{x_{\beta}} \ln \frac{x_{\beta} r}{k_{\mathrm{B}} T k_{\mathrm{off}}}$,

wherein $F$ is the most probable unbinding force, $k_{\mathrm{B}} T=4.114 \mathrm{pN} \mathrm{nm}$ (at $298 \mathrm{~K}$ ) is a Boltzmann factor, $x_{\beta}$ is a molecular length parameter along the reaction coordinate (see below), $r$ is the loading rate, and $k_{\text {off }}$ is the thermal off-rate under zero load. In the lower region $(r<11000 \mathrm{pN} / \mathrm{s})$, the slopes corresponding to the three fragments do not differ within the error margin when this fit is applied to each dataset (see Fig. 4). According to the theoretical model behind the formula used (Evans and Ritchie, 1997; Merkel et al., 1999), this slope can be attributed to the last potential barrier in the energy landscape of the system. In this case, the natural thermal off-rate $k_{\text {off }}$ can be derived by extrapolating the linear fit to the state of zero external force. We obtain a medium off-rate $k_{\text {off }}=(1.2 \pm 1.0) \times 10^{-3} \mathrm{~s}^{-1}$ for all three DNA target sequences, which corresponds to a mean life time of $\tau=(13.9 \pm 11.6) \mathrm{min}$ for the bound protein-DNA complex. The molecular parameter $x_{\beta}$ defines the distance between the minimum of the potential well of the bound state and the maximum of the energy barrier separating the bound state from the free state along the reaction coordinate. This is often interpreted as the depth of the binding pocket (p.e., Merkel et al., 1999). For the lower region, $x_{\beta}=(7.5 \pm 1.0) \AA$ for all proteinDNA complexes can be deduced. 
In the upper region $(r>11000 \mathrm{pN} / \mathrm{s})$, different values for the slopes corresponding to the individual DNA fragments can be found. In accordance with (Merkel et al., 1999), this can be attributed to a second energy barrier in the system, with different properties for the three DNA target sequences. We measure a molecular parameter $x_{\beta}=(2.0 \pm 0.6) \AA$ for the protein-DNA complex with $\exp G 1 / G 4, \quad x_{\beta}=(0.97 \pm 0.06) \AA$ with $\operatorname{expE1/E5}$, and $x_{\beta}=(0.39 \pm 0.14) \AA$ with $\operatorname{expA1/A4}$. We believe these differences to depend on the non-identical nucleotides of the three target sequences in the binding region. It should be noted that the slopes do not increase simply with the length of the DNA fragment, pointing to a sequence-specific mechanism.

\section{Conclusion}

Our experiments demonstrate that ExpG binds to promoter regions in the exp gene cluster in a sequencespecific manner. For the first time, this binding of a regulatory protein-DNA was not only confirmed by standard ensemble methods, but also measured by AFM single molecule force spectroscopy. These experiments yield a thermal off-rate for the dissociation of the bound protein-DNA complex of ca. $10^{-3} \mathrm{~s}^{-1}$ for all four promoter regions. Furthermore, when loading rates $>11000 \mathrm{pN} / \mathrm{s}$ are applied to the bound complex, distinct differences in behavior allow us to distinguish the DNA target sequences of ExpG on a single molecule basis. We suggest that these differences depend on the small number of divergent base pairs within the very palindromic pattern we identified for the binding region by sequence alignment. Interpretation of the molecular parameter $x_{\beta}$ that is connected to these differences remains, however, difficult. Since we obtain exceedingly small $x_{\beta}$ values under high loading rates, it is disputable if these molecular length parameters along the path of separation are directly related to the geometry of the binding mechanism (i.e., the depth of a binding pocket). Further experiments are needed to fully understand the structural information contained in the $x_{\beta}$ parameters: After footprinting experiments to confine the regions of binding more precisely, force spectroscopy on the interaction of ExpG with selected mutants of the DNA target sequences should be able to identify the origin of the $x_{\beta}$ differences. A comparison of our results with kinetic parameters measured in the thermodynamical equilibrium, which could be obtained by different single molecule methods such as fluorescence correlation spectroscopy, would also increase the understanding of the mechanism involved.

Nevertheless, the results of the single molecule experiments are in perfect agreement with those obtained by the established EMSA ensemble technique, demonstrating the equivalence of both methods. Very small quantities of the sample materials were sufficient to perform tests for specificity and off-rate measurements with the AFM, which proves its function as a powerful investigative tool on the molecular scale. Therefore, we propose a combination of single molecule and ensemble methods as a promising approach to enlighten the mechanism of protein-DNA binding.

\section{Acknowledgments}

We thank A. Pühler for helpful discussions. This work was supported by Grant Pu28/17-3 and SFB 613 from Deutsche Forschungsgemeinschaft.

\section{References}

Alekshun, M.N., Levy, S.B., 1999. The mar regulon: multiple resistance to antibiotics and other toxic chemicals. Trends Microbiol. 7, 410-413.

Astete, S.G., Leigh, J.A., 1996. mucS, a gene involved in activation of galactoglucan (EPS II) synthesis gene expression in Rhizobium meliloti. Mol. Plant Microbe Interact. 9, 395-400.

Becker, A., Rüberg, S., Küster, H., Roxlau, A.A., Keller, M., Ivashina, T., Cheng, H.P., Walker, G.C., Pühler, A., 1997. The 32-kilobase exp gene cluster of Rhizobium meliloti directing the biosynthesis of galactoglucan: genetic organization and properties of the encoded gene products. J. Bacteriol. 179, 1375-1384.

Becker, J.D., Moreira, L.M., Kapp, D., Frosch, S.C., Pühler, A., Perlic, A.M., 2001. The nodulin VfENOD18 is an ATP-binding protein in infected cells of Vicia faba L. nodules. Plant Mol. Biol. 47, 749-759.

Bertram-Drogatz, P.A., Quester, I., Becker, A., Pühler, A., 1998. The Sinorhizobium meliloti MucR protein, which is essential for the production of high-molecular-weight succinoglycan exopolysaccharide, binds to short DNA regions upstream of exoH and exo $Y$. Mol. Gen. Genet. 257, 433-441.

Bertram-Drogatz, P.A., Rüberg, S., Becker, A., Pühler, A., 1997. The regulatory protein $M u c R$ binds to a short DNA region located upstream of the mucR coding region in Rhizobium meliloti. Mol. Gen. Genet. 254, 529-538.

Bradford, M.M., 1976. A rapid and sensitive method for the quantitation of microgram quantities of protein utilizing the principle of protein-dye binding. Anal. Biochem. 72, 248-254.

Bustamante, C., Macosko, J.C., Wuite, G.J.L., 2000. Grabbing the cat by the tail: manipulating molecules one by one. Nat. Rev. Mol. Cell Biol. 1, 130-136.

Carrion-Vasquez, M., Oberhauser, A., Fowler, S., Marszalek, P., Broedel, S., Clarke, J., Fernandez, J., 1999. Mechanical and chemical unfolding of a single protein: A comparison. Proc. Natl. Acad. Sci. USA 96, 3694-3699.

Casse, F., Boucher, C., Hulliot, J.S., Michel, M., Dénarié, F., 1979. Identification and characterization of large plasmids in Rhizobium meliloti using agarose gel electrophoresis. J. Bacteriol. 113, 229242

Cohen, S.P., Hachler, H., Levy, S.B., 1993. Genetic and functional analysis of the multiple antibiotic resistance (mar) locus in Escherichia coli. J. Bacteriol. 175, 1484-1492.

Dammer, U., Popescu, O., Wagner, P., Anselmetti, D., Güntherodt, H.-J., Misevic, G.N., 1995. Binding strength between cell adhesion proteoglycans measured by atomic force microscopy. Science 267 , $1173-1175$. 
De Paris, R., Strunz, T., Oroszlan, K., Güntherodt, H.-J., Hegner, M., 2000. Force spectroscopy and dynamics of the biotin-avidin bond studied by scanning force microscopy. Single Molecules 1, 285-290.

Egland, P.G., Harwood, C.S., 1999. BadR, a new MarR family member, regulates anaerobic benzoate degradation by Rhodopseudomonas palustris in concert with AadR, an Fnr family member. J. Bacteriol. 181, 2102-2109.

Evans, E., Ritchie, K., 1997. Dynamic strength of molecular adhesion bonds. Biophys. J. 72, 1541-1555.

Florin, E.-L., Moy, V.T., Gaub, H.E., 1994. Adhesion forces between individual ligand-receptor pairs. Science 264, 415-417.

Fritz, J., Katopodis, A.G., Kolbinger, F., Anselmetti, D., 1998. Forcemediated kinetics of single P-selectin/ligand complexes observed by atomic force microscopy. Proc. Natl. Acad. Sci. USA 95, 12283 12288.

Glazebrook, J., Walker, G.C., 1989. A novel exopolysaccharide can function in place of the calcofluor-binding exopolysaccharide in nodulation of alfalfa by Rhizobium meliloti. Cell 56, 661-672.

Gonzalez, J.E., Reuhs, B.L., Walker, G.C., 1996. Low molecular weight EPS II of Rhizobium meliloti allows nodule invasion in Medicago sativa. Proc. Natl. Acad. Sci. USA 93, 8636-8641.

Gottesman, S., Halpern, E., Trisler, P., 1981. Role of sulA and sulB in filamentation by lon mutants of Escherichia coli K-12. J. Bacteriol. 148, 265-273.

Her, G.R., Glazebrook, J., Walker, G.C., Reinhold, V.N., 1990. Structural studies of a novel exopolysaccharide produced by a mutant of Rhizobium meliloti strain Rm1021. Carbohydr. Res. 198, 305-312.

Hinterdorfer, P., Baumgartner, W., Gruber, H., Schilcher, K., Schindler, H., 1996. Detection and localization of individual antibodyantigen recognition events by atomic force microscopy. Proc. Natl. Acad. Sci. USA 93, 3477-3481.

Hinterdorfer, P., Schilcher, K., Baumgartner, W., Gruber, H., Schindler, H., 1998. A mechanistic study of the dissociation of individual antibody-antigen pairs by atomic force microscopy. Nanobiology 4, 177-188.

Hutter, J.L., Bechhoefer, J., 1993. Calibration of atomic-force microscope tips. Rev. Sci. Instrum. 7, 1868-1873.

Keller, M., Roxlau, A., Weng, W.M., Schmidt, M., Quandt, J., Niehaus, K., Jording, D., Arnold, W., Pühler, A., 1995. Molecular analysis of the Rhizobium meliloti mucR gene regulating the biosynthesis of the exopolysaccharides succinoglycan and galactoglucan. Mol. Plant Microbe Interact. 8, 267-277.

Komeda, H., Kobayashi, M., Shimizu, S., 1996. Characterization of the gene cluster of high-molecular-mass nitrile hydratase $(\mathrm{H}-$ NHase) induced by its reaction product in Rhodococcus rhodochrous J1. Proc. Natl. Acad. Sci. USA 93, 4267-4272.

Lee, G.U., Chrisey, L.A., Colton, R.J., 1994a. Direct measurement of the forces between complementary strands of DNA. Science 266, $771-773$.

Lee, G.U., Kidwell, D.A., Colton, R.J., 1994b. Sensing discrete streptavidin-biotin interactions with atomic force microscopy. Langmuir 10, 354-357.

Lloret, J., Martin, M., Oruezabal, R.I., Bonilla, I., Rivilla, R., 2002. MucR and MucS activate exp genes transcription and galactoglucan production in Sinorhizobium meliloti EFB1. Mol. Plant Microbe Interact. 15, 54-59.

Lloret, J., Wulff, B.B., Rubio, J.M., Downie, J.A., Bonilla, I., Rivilla, R., 1998. Exopolysaccharide II production is regulated by salt in the halotolerant strain Rhizobium meliloti EFB1. Appl. Environ. Microbiol. 64, 1024-1028.
Lyubchenko, Y., Shlyakhtenko, L., Harrington, R., Oden, P., Lindsay, S., 1993. Atomic force microscopy of long DNA: imaging in air and under water. Proc. Natl. Acad. Sci. USA 90, 2137-2140.

Merkel, R., Nassoy, P., Leung, A., Ritchie, K., Evans, E., 1999. Energy landscapes of receptor-ligand bonds explored with dynamic force spectroscopy. Nature 397, 50-53.

Miller, P.F., Sulavik, M.C., 1996. Overlaps and parallels in the regulation of intrinsic multiple-antibiotic resistance in Escherichia coli. Mol. Microbiol. 21, 441-448.

Oscarsson, J., Mizunoe, Y., Uhlin, B.E., Haydon, D.J., 1996. Induction of haemolytic activity in Escherichia coli by the sly $A$ gene product. Mol. Microbiol. 20, 191-199.

Pellock, B.J., Teplitski, M., Boinay, R.P., Bauer, W.D., Walker, G.C., 2002. A LuxR homolog controls production of symbiotically active extracellular polysaccharide II by Sinorhizobium meliloti. J. Bacteriol. 184, 5067-5076.

Rawlins, D.R., Milman, G., Hayward, S.D., Hayward, G.S., 1985. Sequence-specific DNA binding of the Epstein-Barr virus nuclear antigen (EBNA-1) to clustered sites in the plasmid maintenance region. Cell 42, 859-868.

Rief, M., Gautel, M., Oesterhelt, F., Fernandez, J.M., Gaub, H.E., 1997. Reversible unfolding of individual titin immunoglobulin domains by AFM. Science 276, 1109-1112.

Ros, R., Schwesinger, F., Anselmetti, D., Kubon, M., Schäfer, R., Plückthun, A., Tiefenauer, L., 1998. Antigen binding forces of individually addressed single-chain Fv antibody molecules. Proc. Natl. Acad. Sci. USA 95, 7402-7405.

Rüberg, S., Pühler, A., Becker, A., 1999. Biosynthesis of the exopolysaccharide galactoglucan in Sinorhizobium meliloti is subject to a complex control by the phosphate-dependent regulator PhoB and the proteins ExpG and MucR. Microbiology 145, 603611.

Schirmer, F., Ehrt, S., Hillen, W., 1997. Expression, inducer spectrum, domain structure, and function of MopR, the regulator of phenol degradation in Acinetobacter calcoaceticus NCIB8250. J. Bacteriol. $179,1329-1336$

Schwesinger, F., Ros, R., Strunz, T., Anselmetti, D., Güntherodt, H.J., Honegger, A., Jermutus, L., Tiefenauer, L., Plückthun, A., 2000. Unbinding forces of singel antibody-antigen complexes correlate with their thermal dissociation rates. Proc. Natl. Acad. Sci. USA 97, 9972-9977.

Strunz, T., Oroszlan, K., Schäfer, R., Güntherodt, H.-J., 1999. Dynamic force spectroscopy of singel DNA molecules. Proc. Natl. Acad. Sci. USA 96, 11277-11282.

Sulavik, M.C., Gambino, L.F., Miller, P.F., 1995. The MarR repressor of the multiple antibiotic resistance (mar) operon in Escherichia coli: prototypic member of a family of bacterial regulatory proteins involved in sensing phenolic compounds. Mol. Med. 1, 436-446.

Wang, L.X., Wang, Y., Pellock, B., Walker, G.C., 1999. Structural characterization of the symbiotically important low-molecularweight succinoglycan of Sinorhizobium meliloti. J. Bacteriol. 181, 6788-6796.

Zhan, H.J., Lee, C.C., Leigh, J.A., 1991. Induction of the second exopolysaccharide (EPSb) in Rhizobium meliloti SU47 by low phosphate concentrations. J. Bacteriol. 173, 7391-7394.

Zhan, H.J., Levery, S.B., Lee, C.C., Leigh, J.A., 1989. A second exopolysaccharide of Rhizobium meliloti strain SU47 that can function in root nodule invasion. Proc. Natl. Acad. Sci. USA 86, 3055-3059. 